\title{
http://bjas.journals.ekb.eg \\ Association of Lipid Profile and Inflammatory Markers with Coronary Plaque Characteristics Assessed by Multi Slice Coronary CT Angiography in Patients with Coronary Artery Disease
}

H.M.AboulEnein, S.A.Mostafa, H.H.Ebid and A.G.Amer

Cardiology Dept, Faculty of Medicine, Benha Univ., Benha, Egypt

E-Mail:gamalamer 33@yahoo.com

\begin{abstract}
CAD is mainly due to atherosclerosis, an inflammatory process that is based on the interaction between immune mechanisms and metabolic risk factors. Abnormal lipid levels, particularly elevated low-density lipoprotein [LDL-C] and decreased high-density lipoprotein [HDL-C], as well as higher level of inflammatory markers especially high sensitive CRP [hsCRP] are well-established risk factors for cardiovascular disease [CVD].The present work aims to study the relation between lipid profile, high sensitive C-reactive protein[hs-CRP] with coronary plaque characteristics as assessed by Multi slice Coronary CT angiography._Two hundreds and twenty patients presented with typical chest pain were evaluated by MSCT coronary angiography for assessment of calcium score , characters of atherosclerotic plaques as well as identification of vulnerable plaques.Lipid profile and hs-CRP were measured for those patients.
\end{abstract}

Keywords: Atheroscelerosis, Lipid profile, hs-CRP, Vulnerable plaques, MSCT coronary angiography.

\section{Introduction}

Coronary artery disease [CAD], the leading cause of mortality worldwide, places a serious economic burden on health care systems. CAD is mainly due to atherosclerosis, which is the primary cause of mortality and morbidity in cardiovascular disease [CVD]

[1.].Dyslipidemia is a well-established risk factor for the development of coronary artery disease [CAD], and this has been demonstrated in several clinical and epidemiological studies. High plasma low-density lipoprotein [LDL-C] concentrations and low high-density lipoprotein [HDL-C] concentrations have been pointed out as one of the strongest independent risk factors for coronary atherosclerotic disease[2]. Other data suggested that non-HDL cholesterol [non-HDL-C] is a better parameter for assessing CVD risk rather than TC and HDL-C [3]. Also, some studies suggest that lipid ratios, including TC/HDL-C and LDLC/HDL-C ratios are risk factors with better predictive value for coronary atherosclerotic progression or regression than each lipid parameter used independently [4] . Concurrently, C-reactive protein [CRP] has also been proposed as a method of identifying individuals at higher cardiovascular risk. CRP is produced and secreted from the liver and smooth muscle cells surrounding atherosclerotic plaques.[ 5].Studies have demonstrated that CRP correlates with various aspects of atherogenesis as well as significant coronary stenosis [6]. Major advances in CAD prevention require early detection of the vulnerable plaques. A noninvasive assay to directly detect coronary atherosclerosis would therefore be beneficial. Coronary CTA provides comprehensive information noninvasively regarding the location, severity, and characteristics of coronary atherosclerotic plaques [7].

\section{2. patients and methods}

The study will include 220 [ two hundreds and twenty] patients presented to MDCT coronary angiography for evaluation of chest pain. Based on the results of MSCT, we selected 30 patients with normal CTA for the control group. According to WHO diagnostic criteria, the standard CAD group included at least one coronary artery cavity narrowing $\geq 50 \%$ in the left main, left anterior descending [LAD], left circumflex [LCX], or right coronary artery [RCA] as determined by coronary imaging technique. From a group of patients with CAD, we evaluated their CT scales to select 40 patients with soft plaque , 40 patients with mixed plaque, and 40 patients with hard plaque according to the following inclusion and exclusion criteria.

\subsection{Inclusion criteria}

Sinus rhythm., Their heart rate less than 70 bpm spontaneously or Beta blocker induced., They can hold breath for more than 20 seconds., Weight less than $150 \mathrm{~kg}$.

\subsection{Exclusion criteria}

Dye allergy,.,Renal impairment [creatinine >1.5]., Difficulties in performing CT like inadequate breath holding., Previous history of 
coronary invasive maneuvers [PCI or CABG]., Respiratory failure or heart failure, Presence of arrhythmias., Treatment with statin

\subsection{Methodology}

All the patients will be subjected to the following:

*Personal data

*Clinical examination .

*12- Lead ECG.

* Routine lab investigations:

- Serum creatinine.

- ,Lipid Profile

- ,High sensitive C reactive Protein [hsCRP]

- ,Cardiac enzymes to exclude patients with acute coronary syndrome.

\section{* Coronary CT angiography}

The CT angiography will be performed to all patients utilizing a dual source scanner [Somatom Definition Flash, Siemens] with slice configuration of $128 \times 0.625 \mathrm{~mm}$ and gantry rotation time of $330 \mathrm{~ms}$.

2.4 The following parameters will be assessed:

- Coronary calcium score : Based on total calcium score the coronary artery disease is graded

- No evidence of $\mathrm{CAD} \rightarrow 0$ calcium score

- Minimal $\rightarrow$ 1-10

- $\quad$ Mild $\rightarrow$ 11-100

- Moderate $\rightarrow 101-400$

- $\quad$ Severe $\rightarrow>400 \quad$ [8].

- Coronary plaque type and composition.

Using Hounsfield unit three types of plaques will be identified:

*Non-calcified lipid-rich plaques had a density of $\leq 60 \mathrm{HU}$.
*Mixed fibrous plaques had a density of [61 \pm 119$]$

*Calcified plaques had a density of $\geq$ $120 \mathrm{HU}$.

\section{Degree of stenosis}

Obstructive plaques are those plaques showing $50 \%$ stenosis of the arterial lumen or more. [9].

\subsubsection{Signs of vulnerable plaque *Positive Remodeling}

Positive remodeling [outward expansion] is defined as $5 \%$ increase in the luminal crosssection at the site of plaque compared with the normal proximal segment of the vessel.[10] .

\section{*Napkin Ring sign}

The napkin ring sign is defined by inhomogeneous plaque containing a core of lower attenuation material and an outer rim with higher attenuation material. [11].

\subsection{Statistical analysis}

Data will be collected, processed and analyzed using Stastical Package For Social Science [SPSS] version 23 [12].

\section{Results and discussion}

Low density lipoprotein [LDL] from the soft plaque group, mixed plaque group and hard plaque group showed values significantly higher than control group [ $\mathrm{p}=0.001]$

Total cholesterol [TC] from the soft plaque group and mixed plaque group showed values significantly higher than the hard plaque group and control group[ $p=0.00001]$. However total cholesterol in soft plaque showed higher value than mixed plaques but the difference is statistically insignificant $[\mathrm{p}=0.0238]$

High density lipoprotein [HDL] and Total triglycerides [TG] showed no significant difference among the four groups. $[\mathrm{p}=0.467$, $\mathrm{p}=0.991$ respectively].

Table (1) Lipid profile in different plaques

\begin{tabular}{lccccc}
\hline & Soft plaque [40] & $\begin{array}{c}\text { Mixed plaque } \\
{[\mathbf{4 0}]}\end{array}$ & $\begin{array}{c}\text { Hard plaque } \\
{[\mathbf{4 0}]}\end{array}$ & $\begin{array}{c}\text { Control group } \\
{[\mathbf{3 0}]}\end{array}$ & p value \\
\hline $\begin{array}{l}\text { Total } \\
\text { cholesterol }\end{array}$ & $230.5 \pm 23.09$ & $225.5 \pm 12.58$ & $173 \pm 14.62$ & $150.5 \pm 5.77$ & 0.00001 \\
[mg/dl] & & & & & \\
LDL [mg/dl] & $124.52 \pm 40.21$ & $123.36 \pm 42.13$ & $113.30 \pm 33.71$ & $99.77 \pm 28.53$ & 0.001 \\
HDL [mg/dl] & $38.27 \pm 12.8$ & $40.50 \pm 12.668$ & $41.37 \pm 10.27$ & $41.74 \pm 9.29$ & 0.467 \\
Total & $197.25 \pm 68.23$ & $193.98 \pm 63.21$ & $194.86 \pm 41.31$ & $187.75 \pm 26.87$ & 0.991 \\
Triglycerides & & & & & \\
[mg/dl] & & & & & \\
\hline
\end{tabular}

Hs-CRP from the soft plaque group, mixed plaque group and hard plaque group showed values significantly higher than the control group [p<0.001].Also, the mean level of hs$\mathrm{CRP}$ in the soft plaque and mixed plaque 
group was significantly higher than the hard plaque group[p<0.001].However, the difference between the soft plaque group and

Table (2) Hs- CRP in different types ofplaques mixed plaque group was statistically insignificant.[p=0.362]

\begin{tabular}{lcccc}
\hline & Soft plaque [40] & $\begin{array}{c}\text { Mixed plaque } \\
{[\mathbf{4 0 ]}}\end{array}$ & $\begin{array}{c}\text { Hard plaque } \\
{[\mathbf{4 0 ]}}\end{array}$ & $\begin{array}{c}\text { Control group } \\
{[\mathbf{3 0}]}\end{array}$ \\
\hline Hs-CRP $[\mathrm{mg} / \mathrm{L}]$ & $5.21 \pm 1.17$ & $4.91 \pm 1.78$ & $1.71 \pm 1.32$ & $1.44 \pm 1.662$ \\
\hline
\end{tabular}

There were no significant differences in age, sex, smoking, family history of CAD, hypertension, diabetes among the four groups.

Table (3) Effect of other different factors on type of plaque

\begin{tabular}{lccccc}
\hline & $\begin{array}{c}\text { Soft } \\
\text { plaque [40] }\end{array}$ & $\begin{array}{c}\text { Mixed } \\
\text { plaque [40] }\end{array}$ & $\begin{array}{c}\text { Hard } \\
\text { plaque } \\
{[\mathbf{4 0 ]}}\end{array}$ & $\begin{array}{c}\text { Control } \\
\text { group [30] }\end{array}$ & P value \\
\hline Age[years] & $62.9 \pm 10.1$ & $62.3 \pm 10.3$ & $63.6 \pm 6.9$ & $58.4 \pm 6.9$ & 0.108 \\
Sex[male/female, n] & $29 / 11$ & $27 / 13$ & $28 / 12$ & $22 / 8$ & 0.946 \\
Hypertension[n] & 24 & 23 & 25 & 17 & 0.957 \\
Diabetes Mellitus[n] & 25 & 22 & 23 & 17 & 0.917 \\
Family Hx of CAD & 8 & 7 & 6 & 6 & 0.932 \\
Smoking/Nonsmoking & $26 / 14$ & $25 / 15$ & $21 / 19$ & $18 / 12$ & 0.692 \\
[n] & & & & & \\
\hline
\end{tabular}

Some studies have found the diagnostic accuracy of MSCT to be excellent compared with catheter angiography, but there has not been an abundance of clinical outcomes studies. For any new risk marker to be considered useful for risk prediction, it must, at the very least, have an independent statistical association with risk after accounting for established readily available and inexpensive risk markers [13]. CAD is one of the most common causes of death in humans. Recently, its incidence and mortality are increasing. ACS, accounting for $30 \%$ to $40 \%$ of CAD, has been the main cause of CAD patients'poor prognosis and sudden death. Coronary atherosclerosis, plaque formation, plaque instability and disruption, a thrombus in the artery, obliteration of the artery, heart muscle ischemia and even necrosis are the basic pathological mechanisms of CAD. The basic pathology of ACS and sudden coronary death involve vulnerable plaque, vulnerable cardiac muscle, and vulnerable blood [14].

Research shows that almost all the cases of plaque disruption are based on the instability of plaque with little relation to the degree of coronary artery stenosis. A correlation has been shown between elevated systemic inflammation markers, accumulation of inflammatory cells within atherosclerotic soft plaque, and lower fibrous cap thickness [15].Early identification and early intervention with soft vulnerable plaques is definitely important to improving the prognosis in ACS.. We need a non-invasive means to replace early invasive inspection[Coronary angiography amd IVUS] to identify and predict unstable plaque [16] .

Selected biomarkers may be used to predict future cardiovascular events, but the gains over considering conventional risk factors are minimal. Many risk factors have been proposed as predictors of CHD. New risk factors or markers are frequently identified and evaluated as potential additions to standard risk assessment strategies [17].

Histopathology shows that vulnerable plaques are characterized by their connective tissue, a fairly high content of fat and inflammatory masses in soft plaques with the frequent occurrence of hemorrhage, calcification, necrosis and occasional thrombus on the surface. Studies have discovered that CRP is a typical phase synthetic protein in human It is not only a symbol of inflammation but also a direct factor of arterial thrombosis. Serum Hs-CRP levels have a close correlation with inflammation and with the degree of tissue injury. CRP has been shown to participate in the whole atherosclerotic process, including damage of blood vessel endothelium and the formation, maturation, instability and final disruption of atheromatous plaque [18.

We diagnosed all the patients based on their MSCT results.. The results and the levels of serum Hs-CRP were statistically analyzed. The study showed that mean levels of serum HsCRP in the soft plaque group and the mixed plaque group were statistically higher than 
those in the hard plaque group [P $<0.01]$. Mean levels of serum Hs-CRP in the three plaque groups were statistically higher than in the control group $[\mathrm{P}<0.01]$. This agrees with previous research results by Sun et al which suggested that inflammation is involved in the occurrence and development of plaque and is the cause for ACS [19].

Concerning lipid profile, as expected, the majority of the normolipidemia group had almost no affected vessels. In comparison, all dyslipidemia groups except for the hypertriglyceridemia and low HDL patients , were associated with higher rates of coronary artery disease.

An earlier study by Paramsothy et al. showed that, of 4,795 MESA participants, without known clinical cardiovascular disease, those with combined hyperlipidemia, hypercholesterolemia, and MetS had increased relative risk for prevalent CAC compared with normolipidemia participants when adjusting for demographic and CVD risk factors.[20]

Similar to Paramsothy et al., we found that isolated hypertriglyceridemia was not associated with CAC extent [20]. These findings suggest that an isolated elevation in triglyceride levels may not be a pathologic risk factor for subclinical atherosclerosis, although hypertriglyceridemia may still be an important factor in cardiovascular disease.

In contrast to Noda et al. who studied two hundred and eighty-nine patients who underwent 64-slice multidetector CT for suspected coronary artery disease. They found that HDL-c cholesterol levels were more accurate for diagnosing the presence of highrisk coronary plaque[21]. However we found no significant association between low HDL-c and prevalent CAC which is similar to the results obtained by Paramsothy et al..

Further more, elevated LDL-c seems to be the principal determinant of CAC prevalence and its extent, with high TG levels having less influence. This is in line with Paramsothy et al.'s findings where the same dyslipidemia groups were associated with prevalent CAC [22].

\section{Conclusion}

The partial and systemic inflammation has a major effect on arteriosclerosis and its complications. The MSCT coronary angiography can effectually detect coronary atherosclerosis plaque and provide evidence of vulnerable patients. Hs-CRP, indirectly reflect the stabilization degree of plaque and reveal their involvement in the occurrence and development of vulnerable plaques.
Also, Dyslipidemia except for hypertriglyceridemia has an important association with vulnerable atherosclerotic plaques and the development of Coronary artery disease. However the effect of HDL level and its relation with the atherosclerotic process and vulnerability of coronary plaques is still under debate and still need further studies.

\section{References}

[1] D.M.Moreira , R.L.Da Silva , Vieira JL, T.Fattah , ME Lueneberg, and CA Gottschall,Role of vascular inflammation in coronary artery disease, potential of anti-inflammatory drugs in the prevention of atherothrombosis. Inflammation and anti-inflammatory drugs in coronary artery disease. American Journal of Cardiovascular Drugs ,Vol.15,PP.1$11,2015$.

[2] C.W. Tsao and C.J.O Donnell,Lipid Profiles and Coronary Artery Plaque by CT Angiography, Promise for Translation of Biology to Imaging.J Cardiovascular Computed Tomography,Vol.7(2), PP.9193,2013.

[3] Li.Chaoyang ,E.S. Ford , J.Tsai , G.Zhao , L.S.Balluz and S.S.Gidding,Serum Non-high-density lipoprotein cholesterol concentration and risk of death from cardiovascular diseases among U.S. adults with diagnosed diabetes: the Third National Health and Nutrition Examination Survey linked mortality study . Cardiovascular Diabetology ,Vol.10,PP.46- 57,2011.

[4] T.Kimura, T.Itoh, T.Fusazaki , H.Matsui ,S. Sugawara , Y.Ogin , H.Endo, K.Kobayashi and M.Nakamura,Lowdensity lipoprotein-cholesterol / highdensity lipoprotein-cholesterol ratio predicts lipid-rich coronary plaque in patients with coronary artery disease: Integrated-backscatter intravascular ultra sound study. Circ J, Vol. 74, PP.1392 $1398,2010$.

[5] M. A. Rahman, A. S. Islam,J. K. Saha ,M. Abul Quashem, Preoperative High Sensitivity C-reactive Protein Level Predicts Early Outcome After Coronary Artery By-pass Surgery. KYAMC Journal, Vol.9(4),pp153-158,2019.

[6] J.Rubin, H.J. Chang , K. Nasir ,Association between high-sensitivity Creactive protein and coronary plaque subtypes assessed by 64-slice coronary computed tomography angiography in an asymptomatic population," Circulation: 
Cardiovascular Imaging, Vol.4 (3) ,PP.201-209,2011.

[7] Z.G. Chu , Z.G.Yang , Z.H. Dong, Characteristics of coronary artery disease in symptomatic type 2 diabetic patients: evaluation with CT angiography," Cardiovascular Diabetology, Vol.9 (1) ,PP.74-77,2010

[8] N. van der Bijl, R.M.Joemai, J.Geleijns, Assessment of Agatston coronary artery calcium score using contrast-enhanced CT coronary angiography. AJR Am J Roentgenol,Vol.195(6), PP.1299305,2010

[9] Arab-Zadeh and J.Hoe, Quantification of coronary arterial stenoses by multidetector CT angiography in comparison with conventional angiography: methods, caveats, and implications. JACC: Cardiovascular Imaging,Vol.4(2), PP.191202,2011.

[10] Y.J Hong, M.H.Jeong , Y.H.C. SukKo , M.G.Lee , W. Won YuKang, Positive remodeling is associated with more plaque vulnerability and higher frequency of plaque prolapse accompanied with postprocedural cardiac enzyme elevation compared with intermediate/negative remodeling in patients with acute myocardial infarction. J Cardiol,Vol.53(2), PP.278-287,2009.

[11] K.Otsuka ， S.Fukuda , A.Tanaka , K. Nakanishi , H.Taguchi , J.Yoshikawa , K.Shimada , M.Yoshiyama ,Napkin-ring sign on coronary CT angiography for the prediction of acute coronary syndrome. JACC Cardiovasc Imaging ,Vol.6(4), PP.448-57,2013.

[12] R.Winters, A.Winters and R.G. Amedee, Statistics: A Brief Overview. The Ochsner Journal,Vol.10, PP.213$216,2010$.

[13]P.Greenland ， J.S.Alpert, G.A.Beller, E.J.Benjamin, M.J.Budoff, Z.A.Fayad, 2010 ACCF/AHA guideline for assessment of cardiovascular risk in asymptomatic adults. J Am Coll Cardiol ,Vol. 56, PP.e50-e103,2010.

[14] M. Laimoud, F. Faris, and H. Elghawaby, Coronary Atherosclerotic Plaque Vulnerability Rather than Stenosis Predisposes to Non-ST Elevation Acute Coronary Syndromes. Cardiol Res
Pract.Article ID

,Vol.12(3),PP.23-31,2019.

2642740

[15]P. Poredos, A. Spirkoska, L. Lezaic, M. B. Mijovski, and M. K. Jezovnik, Patients with an Inflamed Atherosclerotic Plaque have Increased Levels of Circulating Inflammatory Markers. J Atheroscler Thromb,Vol.24(1), PP.39-46,2017.

[16]M.K.Hong ， G.S.Mintz ， C.W.Lee , Y.H.Kim, S.W.Lee, J.M.Song, Comparison of coronary plaque rupture between stable angina and acute myocardial infarction. Circulation,Vol.110,PP.928-933,2004.

[17]P.M.Ridker ， N.P.Paynter ， N.Rifai , J.M.Gaziano , N.R.Cook ,C-reactive protein and parental history improve global cardiovascular risk prediction: the Reynolds Risk Score for men. Circulation,Vol.118,PP.2243-2251,2002

[18][18] P.M.Ridker , N.P.Paynter , N.Rifai , J.M.Gaziano ， N.R.Cook , C-reactive protein and parental history improve global cardiovascular risk prediction: the Reynolds Risk Score for men. Circulation ,Vol.118,PP.2243-2251,2008.

[19]Z. Sun, C. Lin , R. Davidson , C. Donq , Y.Liao, Diagnostic value of 64-slice CT angiography in coronary artery disease: a systematic review. Eur J Radiol,Vol. 67,PP.78-84,2008.

[20]P. Paramsothy, R. H. Knopp, A. G. Bertoni ,"Association of combinations of lipid parameters with carotid intima-media thickness and coronary artery calcium in the MESA [Multi-Ethnic Study of Atherosclerosis]," Journal of the American College of Cardiology, Vol. 56, PP.10341041, 2010

[21] Y. Noda, R. Matsutera, Y. Kohama "Impact of coronary calcium score on the relation between high-density lipoprotein cholesterol levels and the presence of high-risk coronary plaque detected by coronary computed tomography angiography," Journal of the American College of Cardiology, Vol. 63,PP.10641069, 2014.

[22] C. W. Tsao, S. R. Preis, G. M. Peloso "Relations of long-term and contemporary lipid levels and lipid genetic risk scores with coronary artery calcium in the Framingham Heart Study," Journal of the American College of Cardiology, Vol. 60, PP.2364-2371, 2012. 